\title{
Prefrontal Cortex Deep Brain Stimulation Improves Fear and Anxiety-Like Behavior and Reduces Basolateral Amygdala Activity in a Preclinical Model of Posttraumatic Stress Disorder
}

\author{
Roman Reznikov', Francis Rodriguez Bambico',2, Mustansir Diwan', Roger J Raymond', Mina G Nashed', \\ José N Nobrega ${ }^{1,3}$ and Clement Hamani, ${ }^{*, 1,3,4}$ \\ 'Behavioural Neurobiology Laboratory, Research Imaging Centre, Centre for Addiction and Mental Health, Toronto, ON, Canada; ${ }^{2}$ Department of \\ Psychology, Memorial University of Newfoundland, St John's, NL, Canada; ${ }^{3}$ Campbell Family Mental Health Research Institute, Centre for Addiction \\ and Mental Health, Toronto, ON, Canada; ${ }^{4}$ Division of Neurosurgery, Sunnybrook Health Sciences Centre, Toronto, ON, Canada
}

\begin{abstract}
Deep brain stimulation (DBS) is being investigated for a number of psychiatric indications, including posttraumatic stress disorder (PTSD). Preclinical studies continue to be a cornerstone for the development of new DBS applications. We investigate whether DBS delivered to the infralimbic cortex $(\mathrm{IL})$, a region involved in mechanisms of stress resiliency, may counter behavioral abnormalities in rats that present persistent extinction deficits and long-term anxiety after exposure to fear conditioning. Rats undergoing fear conditioning/extinction were segregated into weak and strong extinction groups (WE $>70 \%$ or SE $<30 \%$ of freezing during extinction). Following 2 weeks of DBS, animals were exposed to novel recall sessions and tested in the open field, novelty-suppressed feeding, and elevated plus maze. zif268 expression was measured in structures involved in mechanisms of fear and stress. In vivo electrophysiology was used to record activity from the basolateral amygdala (BLA). We found that DBS improved extinction deficits and anxiety-like behavior in WE animals, having no significant effects in SE rats. No major differences in absolute zif268 levels were recorded across groups. However, correlation between zif268 expression in the IL and BLA was disrupted in WE animals, a deficit that was countered by DBS treatment. Electrophysiology experiments have shown that DBS reduced BLA firing of both putative principal cells and interneurons in WE rats, with no significant differences being detected between SE and SE DBS animals. In summary, IL DBS mitigated fear, partially improved anxiety-like behavior, reversed neurocircuitry abnormalities, and reduced BLA cell firing in a preclinical model of PTSD.
\end{abstract}

Neuropsychopharmacology (2018) 43, I099-I 106; doi:I0.1038/npp.2017.207; published online I5 November 2017

\section{INTRODUCTION}

It is estimated that $50-60 \%$ of the American population will be exposed to at least one lifetime traumatic event (Breslau et al, 1991, 1998; Fairbank et al, 2000; Kessler et al, 1995). Though most individuals recover from the experience, $\sim 10-$ $30 \%$ develop posttraumatic stress disorder (PTSD). Although medications and psychotherapy are often effective, $20-30 \%$ of patients do not respond to conventional therapies. Deep brain stimulation (DBS) has been investigated for various psychiatric indications and is approved for the treatment of obsessive-compulsive disorder. To date, only one patient with PTSD treated with DBS has been reported (Langevin et al, 2016).

Although not without limitations, preclinical studies continue to be a cornerstone of translational research and

\footnotetext{
* Correspondence: Professor C Hamani, Behavioural Neurobiology Laboratory, Centre for Addiction and Mental Health, 250 College Street, Toronto, ON M5T IR8, Canada, Tel: + I 4l6 603577I, E-mail: Clement.Hamani@camh.ca

Received 28 June 2017; revised 6 August 2017; accepted 29 August 2017; accepted article preview online I September 2017
}

development of new treatment approaches (Hamani and Temel, 2012). Rodent subpopulations with maladaptive stress responses have been used by different investigators to mimic PTSD-like states (Bush et al, 2007; Cohen et al, 2004). Similar to PTSD, we have found that $\sim 20-30 \%$ of outbred rats show impaired fear extinction after undergoing stress (Reznikov et al, 2015). These rats, which we refer to as 'weak extinction' (WE), also develop long-lasting anxiety traits and have low baseline corticosterone levels (Reznikov et al, 2015), a feature that is commonly reported in PTSD (Yehuda et al, 1990). Being an eminently clinical condition, some PTSD symptoms cannot be modeled in animals. However, WE rats seem to be suitable for studying long-term fear and anxiety responses that develop following a traumatic experience (Reznikov et al, 2015).

The ventromedial prefrontal cortex, particularly the infralimbic cortex (IL), acts as an essential component for the extinction of conditioned fear responses. Whereas the pharmacological inactivation of IL impairs extinction learning and recall in rodents (Sierra-Mercado et al, 2011), IL stimulation enhances extinction and decreases fear expression (Maroun et al, 2012; Milad and Quirk, 2002; 
Vidal-Gonzalez et al, 2006). These latter effects, however, have only been reported when brief trains of current were coupled with conditioned stimuli (Milad and Quirk, 2002; Vidal-Gonzalez et al, 2006) or when more sustained stimulation (eg, $10 \mathrm{~min}$ ) was administered immediately after the retrieval of a fear memory (Maroun et al, 2012). This is in contrast to the chronic use of DBS in humans who receive stimulation over long periods of time.

One of the consequences of applying short trains of stimulation to the rodent prefrontal cortex is the decrease in firing rate of basolateral amygdala (BLA) principal cells (Likhtik et al, 2005; Rosenkranz and Grace, 2001; Rosenkranz et al, 2003). This is of importance as enhanced BLA activity may facilitate the encoding of fear memories, impair fear extinction, and contribute to enhance anxiety (Maren, 2003; Maren and Quirk, 2004; Reznikov et al, 2016).

In this study we test the hypothesis that continuous IL DBS reduces fear expression and anxiety-like responses in $\mathrm{WE}$ rats while modulating the neurocircuitry of fear and anxiety.

\section{MATERIALS AND METHODS}

Protocols were approved by the animal care committee of the Centre for Addiction and Mental Health and are in accordance with the Canadian Council on Animal Care (CCAC) guidelines. Adult male Sprague-Dawley rats (250300 g; Charles River, Quebec) were used. A timeline for the experiments is shown in Supplementary Figure 1.

\section{Fear Conditioning and Extinction}

On day 1, rats were presented with six conditioned stimuli (CS; $30 \mathrm{~s}, 85 \mathrm{~dB}, 4 \mathrm{hz}$ auditory tones), each co-terminating with a footshock (unconditioned stimulus (US); $0.8 \mathrm{~mA}$, $0.5 \mathrm{~s}$ ) (Reznikov et al, 2015). On day 2, rats underwent extinction training consisting of 12 presentations of the CS in the absence of shocks. On day 3, rats were exposed to 3 presentations of the CS alone to test for extinction recall. All trials were recorded with a video camera for offline scoring of freezing behavior by a blinded investigator.

Segregation of subgroups according to freezing scores during the last extinction blocks (4 trials) was conducted as previously described (Reznikov et al, 2015). Weak extinction (WE) and strong extinction (SE) animals were defined as those showing $>70 \%$ and $<30 \%$ freezing, respectively (Reznikov et al, 2015). Animals with moderate levels of freezing were not included in this study.

\section{Electrode Implantation and DBS}

At 1 week before behavioral experiments (acute DBS experiments) or 1 day following recall sessions (chronic DBS experiments), rats were anesthetized with isofluorane and had insulated stainless steel electrodes (cathodes; $250 \mu \mathrm{m}$ diameter with $0.5 \mathrm{~mm}$ of surface exposed) bilaterally implanted into the IL (anteroposterior +3.0, lateral \pm 0.4 , and depth $5.6 \mathrm{~mm}$ ) (Paxinos and Watson, 1988). Stainless steel screws implanted over the parietal cortex were used as anodes (Paxinos and Watson, 1988). Controls (CTL) were anesthetized, had burr holes drilled into the skull but were not implanted with electrodes. Shams had electrodes implanted in the IL but did not receive DBS.
DBS was conducted with an ANS stimulator (model 3510, St Jude Medical, Plano, TX), connected to the animals through extension cables and a multichannel commutator (Hamani et al, 2010a, b, c, 2012a). Acute DBS was delivered following conditioning, prior and/or during extinction at different settings ( 100 or $300 \mu \mathrm{A}, 90 \mu \mathrm{s}, 10$ or $130 \mathrm{~Hz}$ ). These were chosen based on efficacy in previous studies (Milad et al, 2004; Vidal-Gonzalez et al, 2006) and because they generate a charge density that is similar to that used in patients receiving DBS (Hamani et al, 2010a, b). Chronic DBS was commenced 1 week after electrode implantation and given to the animals for $8 \mathrm{~h}$ /day until the end of the experiments at $100 \mu \mathrm{A}, 90 \mu \mathrm{s}$, and $130 \mathrm{~Hz}$. On behavioral testing days, DBS was delivered for $2 \mathrm{~h}$ before and $4 \mathrm{~h}$ after the assessments.

\section{Behavioral Tests}

Extinction recall. Long-term postoperative recall sessions were carried out before DBS was commenced and on days 4, 8 , and 14 after DBS. These consisted of reexposing rats to three presentations of the CS alone.

Open field. On DBS day 16, locomotor activity was assessed for $30 \mathrm{~min}$ in a square $0.49 \mathrm{~m}^{2}$ open field box (Med Associates, St Albans, VT) with infrared photo beams placed along the walls of the equipment. Crossing of the beams provided counts of locomotor activity.

Novelty suppressed feeding. On DBS day 18, rats were placed in a Plexiglas cage (Med Associates) lined with black card material on all sides and bottom that contained a white platform with a previously habituated treat on top. Latency to begin consuming the food was measured by a blinded observer (sniffing or simply touching the food was not scored).

Elevated plus maze. On DBS day 20, rats were placed in the center of the maze and allowed to explore for $5 \mathrm{~min}$. Behavior was recorded by a vertically mounted video camera and later scored for the number of entries and the total time spent in open arms (Reznikov et al, 2015).

\section{In Situ Hybridization and Histology}

Following sacrifice, brains were removed from the skull. Coronal sections were cut on a cryostat and hybridized with ${ }^{35}$ S-UTP-labeled riboprobes complementary to zif268, as previously described (Creed et al, 2012). After hybridization, slides were exposed to Kodak BioMax film for 6 days at $4{ }^{\circ} \mathrm{C}$ along with calibrated radioactivity standards. Film analyses were conducted with an MCID system (Interfocus, Cambridge, UK). Electrode placement was confirmed in cresyl violet-stained sections (Hamani et al, 2012a).

\section{Electrophysiology}

Animals undergoing electrophysiology were distinct from those used in behavioral experiments. Following fear conditioning, extinction, and recall, rats were subdivided into WE and SE. Groups were then subdivided into those to receive active or no stimulation for 2 weeks, followed by a 
new recall session. On the next day, rats were anesthetized with urethane $(1.5 \mathrm{~g} / \mathrm{kg}$ i.p.) and BLA recordings were carried out using single-barreled glass micropipettes filled with $2 \mathrm{M} \mathrm{NaCl}(1-3 \mu \mathrm{m}$ in diameter, 2-6 M $\Omega$ ). Animals were not given DBS on the day of the electrophysiology experiments.

To maximize sampling without introducing excessive tissue damage, three to five electrode descents were performed per hemisphere. Single-unit activity was recorded for at least $5 \mathrm{~min}$. At the end of each recording session, the brain was extracted for histological verification of electrode descents and recording sites. The identity of BLA neurons was ascertained based on the electrophysiological criteria proposed by Likhtik et al (2006) and by Rosenkranz and Grace (1999). Three clusters of neurons were analyzed: low firing $(\leqslant 1 \mathrm{~Hz})$ long-duration signals $(\geqslant 0.7 \mathrm{~ms}$ cutoff) suggested to characterize putative principal projection cells; high firing short-duration cells ( $\leqslant 0.5 \mathrm{~ms}$ cutoff), suggestive of putative fast-spiking interneurons; and neurons with firing rates between 1 and $7 \mathrm{~Hz}$ that are often unclassified or suggestive of medium-firing neurons. As we found a relatively small number of cells firing $>1 \mathrm{~Hz}$ and principal cells often fire below this threshold, BLA neurons in our study were simply classified as $<1 \mathrm{~Hz}$ or $>1 \mathrm{~Hz}$.

\section{Statistical Analyses}

For acute behavioral experiments, repeated measures ANOVA was used to compare fear conditioning/extinction results over time. Recall scores were compared using Student's $t$-test. Behavioral data in chronic DBS experiments and zif268 data were analyzed with two-way ANOVAs (LSD post hoc) using DBS and group (WE/SE) as factors. The following groups were considered WE CTL, SE CTL, WE DBS, and SE DBS. Pearson's correlation was used to measure covariations in the expression of zif268 across brain regions. Correlations were considered to be strong, moderate, or weak when $r \geqslant 0.75,0.75<r \leqslant 0.50$, or $0.50<r \leqslant 0.25$, respectively. Electrophysiology data did not follow a normal distribution. For this reason, it was analyzed with the Kruskall-Wallis test (Dunn's post hoc). Percentages were compared with the $\chi^{2}$ test. Results are reported as mean $\pm \mathrm{SE}$ or median and quartiles, when indicated.

\section{RESULTS}

\section{Behavioral Effects of DBS}

In a first series of experiments, IL-DBS was given to different groups of animals before and during extinction at 100 or $300 \mu \mathrm{A}, 90 \mu \mathrm{s}$, and either $10 \mathrm{~Hz}$ or $130 \mathrm{~Hz}(n=6-10$ /group) (Supplementary Figure 2). Overall, no differences in freezing scores were recorded during recall sessions when stimulated groups were compared with controls (Supplementary Table 1).

Following these negative findings, we decided to test whether chronic IL stimulation could improve long-term fear and anxiety responses in WE rats. After undergoing fear conditioning, extinction, and recall sessions, animals were subdivided into $\mathrm{WE}$ and $\mathrm{SE}$ groups (time $\times$ group interaction during extinction $\mathrm{F}(5,190)=23.6, p<0.001$; group effect $\mathrm{F}$ $(1,38)=112.3, \quad p<0.001 ; \quad$ time effect $\mathrm{F}(5,190)=3.42$,
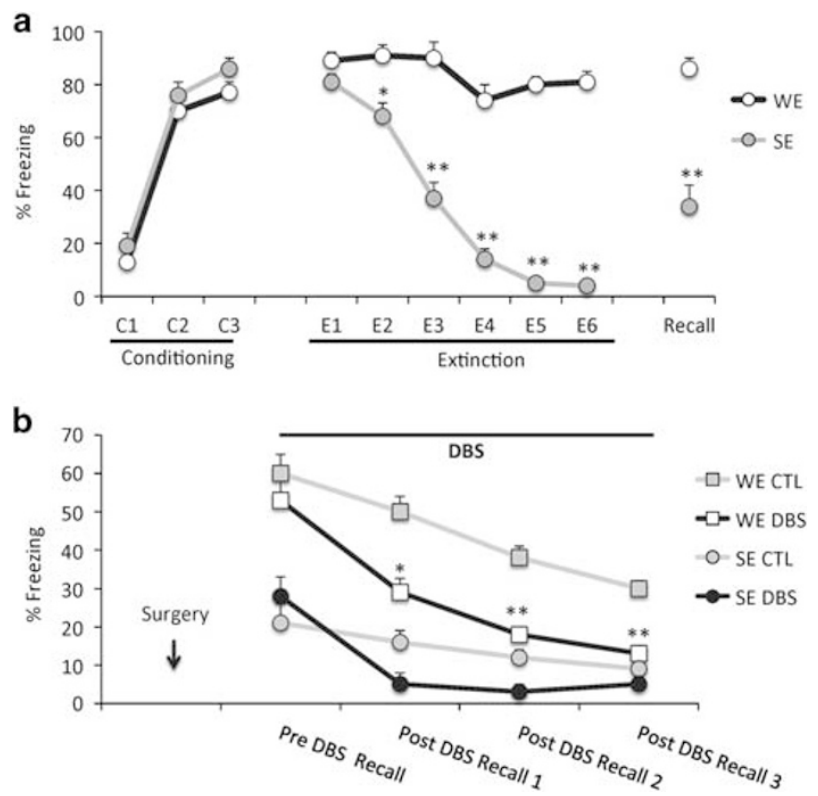

Figure I Chronic deep brain stimulation (DBS) improves freezing in extinction recall trials in weak extinction rats. (a) Fear conditioning, extinction, and short-term recall trials were conducted over three consecutive days. Animals were then separated in weak extinction (WE) or strong extinction (SE) populations. As can be appreciated, during the last four extinction trials (Extinction) and short-term recall, freezing was significantly higher in WE than in SE rats ( $p \leqslant 0.05$; ** $p \leqslant 0.01$ ). Thereafter, animals were implanted with electrodes and I week later submitted to a pre-DBS recall session. (b) Although the distinction in freezing between SE $(n=10)$ and WE animals $(n=9)$ persisted, no significant differences were observed in animals that did and did not receive infralimbic cortex (IL) electrode implants. Following this session, DBS was delivered to the animals for $8 \mathrm{~h} /$ day at $100 \mu \mathrm{A}, 90 \mu \mathrm{s}$, and $130 \mathrm{~Hz}$. New recall sessions were conducted on days 4, 8 , and I 4 after DBS (recall I, 2, and 3, respectively, after DBS). In the former, a significant reduction in freezing was already noticeable in WE rats given DBS $(n=11)$. This became even more pronounced on days 8 and 14 after DBS, with freezing scores in the WE DBS group resembling those observed in SE rats. In contrast, DBS did not significantly affect behavioral scores in SE animals $(n=10)$; $* p \leqslant 0.05$, ** $p \leqslant 0.01$ when WE CTL and WE DBS groups were compared. Significant differences between WE CTL and SE CTL or WE CTL and SE DBS were not represented in the figure for clarity.

$p<0.001$; Figure 1a). The next day, they were implanted with IL electrodes and allowed 1 week to recover (Reznikov et al, 2015). A second recall session conducted at this point confirmed that freezing scores were significantly higher in WE than in SE groups $(\mathrm{F}(1,37)=24.7, p<0.001)$. No effect of electrode insertion $(\mathrm{F}(1,37)=0.02, p=0.89)$ or electrode $\times$ group interaction $(\mathrm{F}(1,37)=0.89, p=0.35)$ were noticed. Thereafter, animals were given daily DBS, with recall sessions being conducted on stimulation days 4, 8, and 14 . Similar behavioral responses were recorded at these three time points. The number of animals per group was as follows: WE CTL $(n=9)$, SE CTL $(n=10)$, WE DBS $(n=11)$, and SE DBS $(n=10)$.

Significant group $(\mathrm{F}(1,37)=18.63, p=0.0001)$ and DBS effects $(\mathrm{F}(1,37)=5.87, p=0.02)$ but no DBS $\times$ group interaction $(\mathrm{F}(1,37)=0.52 ; p=0.47)$ were found on day 4 recall sessions after DBS. Freezing scores in WE DBS animals $(29.3 \pm 7.1 \%)$ were lower than in non-stimulated WE-CTL 
$(50.2 \pm 10.3 \% ; p=0.03)$. In contrast, DBS was not found to be significantly effective in SE rats (Figure 1b).

On poststimulation day 8 , group effect $(\mathrm{F}(1,37)=25.9$, $p<0.001)$, DBS effect $(\mathrm{F}(1,37)=17.3, p=0.0002)$ and group $\times$ DBS interaction $(\mathrm{F}(1,37)=4.5 ; p=0.04)$ were all found to be significant. At this interval, rats in the WE-DBS group $(14.5 \pm 3.1 \%)$ froze significantly less than WE-CTL rats $(38.3 \pm 6.8 \%, p<0.001$; Figure $1 b)$. No significant behavioral differences were found between stimulated and nonstimulated SE rats.

After 14 days of stimulation, significant group $(\mathrm{F}(1,36)=12.0$, $p=0.0014)$ and DBS effects $(\mathrm{F}(1,36)=6.3, p=0.02)$ were recorded with a trend toward a significant group $\times$ DBS interaction $(\mathrm{F}(1,36)=3.5, p=0.07)$. As noticed during other time points, DBS-treated WE rats froze less $(12.0 \pm 2.8 \%)$ than WE-CTL $(29.6 \pm 7.7 \%, p=0.005)$ with no significant differences being detected between stimulated $(5.6 \pm 2.3 \%)$ and nonstimulated SE groups $(8.2 \pm 1.8 \%, p=0.6$; Figure $1 b)$.

Locomotion. To assess whether DBS-induced changes in freezing could be attributed to a general locomotor effect, exploratory activity was tested in the open field. Ruling out this possibility, no significant effects of group $(\mathrm{F}(1,31)=1.1$, $p=0.30)$, DBS $(\mathrm{F}(1,31)=0.007, p=0.93)$, or group $\times \mathrm{DBS}$ interaction were recorded $(\mathrm{F}(1,31)=1.8, p=0.19$; Figure $2 \mathrm{a})$.

Anxiety-like responses. To study the effects of DBS on long-term anxiety responses, WE and SE rats given chronic stimulation were tested in a novelty suppressed feeding paradigm and an elevated plus maze. In the former, two-way
ANOVA revealed significant DBS $(\mathrm{F}(1,31)=4.7, p=0.04)$ and group effects $(\mathrm{F}(1,31)=10.3, p=0.003)$ but no significant $\mathrm{DBS} \times$ group interaction $(\mathrm{F}(1,31)=2.1, p=0.2)$. Latency to eat in an open arena was significantly lower in SE $(151.9 \pm 19.7 \mathrm{~s})$ than in WE rats $(347.7 \pm 57.8 \mathrm{~s}, p=0001)$. DBS treatment reduced latency to feed in WE animals $(195.0 \pm 46.1 \mathrm{~s}, p=0.01$ as compared with WE CTL) having no significant effects in SE rats $(121.3 \pm 26.5 \mathrm{~s}, p=0.6$ as compared with SE-CTL; Figure 2b).

In the elevated plus maze, two-way ANOVA for total entries and time spent in open arms of the elevated plus maze did not show a significant DBS effect $\left(\mathrm{F}_{\text {entries }}(1,31)=0.5, p=0.50\right.$; $\left.\mathrm{F}_{\text {time }}(1,31)=0.9, p=0.36\right)$, group effect $\left(\mathrm{F}_{\text {entries }}(1,31)=0.007\right.$, $\left.p=0.94 ; F_{\text {time }}(1,30)=0.15, p=0.7\right)$, or a positive $\mathrm{DBS} \times$ group interaction $\quad\left(\mathrm{F}_{\text {entries }}(1,31)=2.9 \quad p=0.1 ; \quad \mathrm{F}_{\text {time }}(1,31)=3.1\right.$, $p=0.09)$. Mean values in the WE DBS group, however, were approximately twice as high as those recorded in nonstimulated WE controls (entries 5.4 vs 3.0; time 42.7 vs $19.7 \mathrm{~s}$; Figure 2c).

To test the behavioral effects of electrode insertion, we have implanted a separate group of WE rats with IL electrodes. In contrast to DBS results, no significant differences were found between shams $(n=6)$ and nonimplanted controls ( $n=6$; Supplementary Figure 3).

\section{Immediate Early Gene Expression}

To characterize DBS-induced neurocircuitry changes in SE $(n=8)$ and WE rats $(n=6)$, zif268 expression was measured
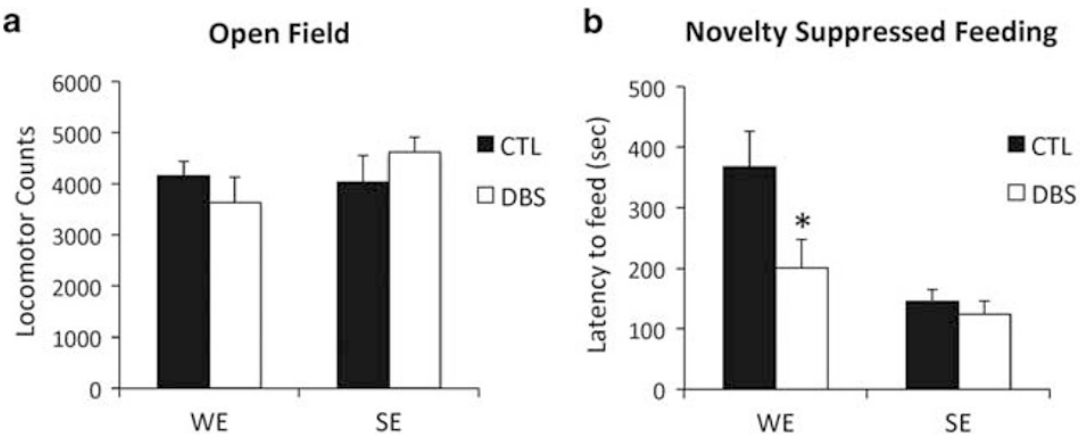

\section{Elevated Plus Maze}
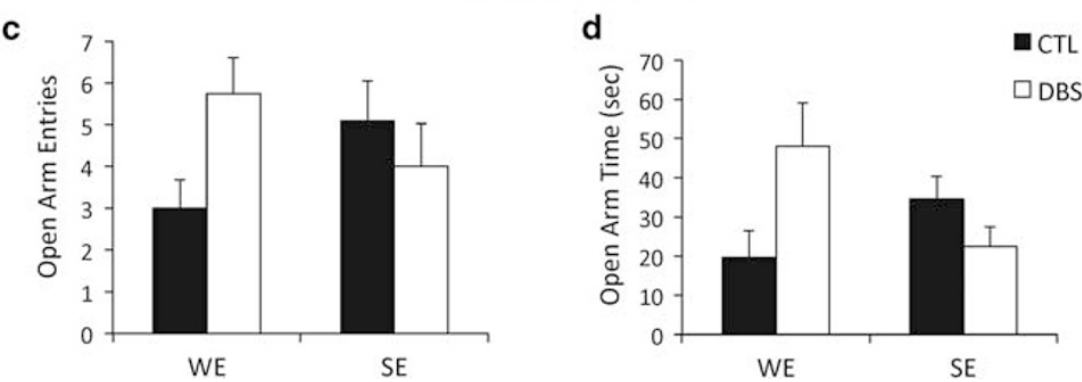

Figure 2 Antianxiety effects of infralimbic cortex (IL) deep brain stimulation (DBS) in weak extinction (WE) rats. On poststimulation days I6, I8, and 20, animals were tested in the open field, novelty suppressed feeding test (NSF), and elevated plus maze (EPM). (a) In the open field, no differences across groups were found in exploratory behavior, as revealed by similar locomotive counts. (b) In the NSF, WE-CTL took significantly longer to consume a habituated treat in a novel environment than SE-CTL. DBS significantly decreased this latency in WE animals but had no effect in the SE group. (c) In the EPM, the number entries and (d) the time WE animals spent in the open arms of the maze were almost twice as low as in SE-CTL. These differences, however, did not reach statistical significance. DBS reversed these deficits with a trend toward an increase in the number of entries and an increase in the time spent in the open arms of the maze. In all figures, values are means \pm SEM. $* P \leqslant 0.05$ when WE-CTL and WE DBS groups were compared. WE CTL ( $n=8$ rats), WE DBS ( $n=8$ rats), SE CTL ( $n=9$ rats), and SE DBS ( $n=9$ rats). 
in structures involved in mechanisms of fear and anxiety. These included the PFC (IL, prelimbic cortex, cingulate cortex), amygdala (lateral nucleus, basolateral nucleus, and central nucleus), and hippocampus (granule cell layer of the dentate gyrus, pyramidal cell layer of CA3 and CA1). Overall, the only significant finding was a DBS effect $(\mathrm{F}(1,23)=6.21$, $p=0.02$ ) in the CA1 region (Figure 3 and Supplementary Table 2). In both SE and WE rats, a significant reduction in zif268 levels was found in stimulated animals.

In a second series of analyses, we studied the correlation between zif268 expression in the target structure (ie, IL) and each of the above-described regions. In the prefrontal cortex, strong positive correlations were found between IL and PL, IL and Cg1, and IL and Cg2 in almost all groups (Table 1). The only exception was the somewhat lower correlation between IL-Cg1 in WE rats, a pattern that was reversed by DBS.

In the amygdala, weak, moderate, or strong positive correlations were found between IL and La and IL and Ce across groups (Table 1). In the BLA, however, although a weak positive correlation with IL was found in SE CTL, negative values were recorded in the WE CTL group (Table 1). Despite not being strong, it was the only instance in which correlations were found to be inverted in WE and SE CTLs. DBS reversed this pattern, with positive correlations being recorded in WE stimulated rats while having no major effect in the SE group.

Correlations between IL and hippocampal DG zif268 levels were similar in SE and WE animals. In both groups, these were substantially reduced by DBS (Table 1). IL-CA1 and ILCA3 correlations were similar across groups.

\section{Electrophysiology}

As DBS was found to counter the altered correlation of ILBLA zif268 expression in WE rats, we decided to study the effects of chronic stimulation on BLA firing using in vivo electrophysiology.

Thirty-six cells were recorded from WE CTL rats $(n=4)$, 48 from WE animals given chronic DBS $(n=4), 45$ from the SE CTL group $(n=4)$, and 31 from SE DBS rats $(n=3)$. When all cells were considered, differences in firing across groups were found to be significant $(p=0.02)$. Overall, BLA neurons in WE CTL $(1.33 \pm 0.48 \mathrm{~Hz}$; median 0.41$)$ fired at higher rates than in WE DBS $(0.33 \pm 0.07$; median 0.08 ; mean rank diff. $=33.9)$, SE DBS $(0.23 \pm 0.09$; median 0.06 ; mean rank diff. $=45.9)$, and, to a lesser extent, SE CTL $(0.45 \pm 0.12$; median 0.14; Figure 4a).

In WE CTL rats, $22 \%(8 / 38)$ of BLA cells fired $>1 \mathrm{~Hz}$. Although this proportion was 2-3 times higher than in SE CTL $(9 \% ; 4 / 45)$, WE DBS $(8 \% ; 4 / 48)$, and SE DBS groups $(6 \% ; 2 / 31)$, results did not reach statistical significance $(p=0.29$; Figure $4 \mathrm{~b})$. Similarly, the firing rate of $>1 \mathrm{~Hz}$ cells was also twice as high in WE CTL $(4.9 \pm 1.7 \mathrm{~Hz}$; median 1.8$)$ than in SE CTL $(2.2 \pm 0.5 \mathrm{~Hz}$; median 2.3), WE DBS $(1.8 \pm 0.2 \mathrm{~Hz}$; median 1.9), and SE DBS groups $(1.8 \pm 0.7 \mathrm{~Hz}$; median 1.8$)$. These results were also not statistically significant ( $p=0.97$; Figure $4 c)$.

In contrast to these findings, significant differences in firing across groups were found when $<1 \mathrm{~Hz}$ BLA cells were examined $(p<0.01$; Figure $4 \mathrm{~d})$. Although WE CTL $(0.3 \pm 0.04 \mathrm{~Hz} ;$ median 0.25$)$ and SE CTL animals

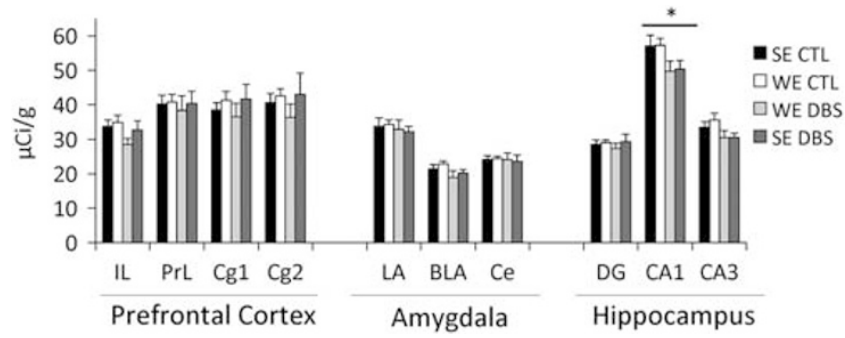

Figure 3 zif268 expression in the prefrontal cortex, amygdala, and hippocampus of animals treated with chronic infralimbic cortex (IL) deep brain stimulation (DBS). Two-way ANOVA revealed a significant DBS effect in the CAI region (* $p \leqslant 0.05$ ) with no significant differences being detected between stimulated animals and nonstimulated controls in post hoc analyses. In the remaining regions, differences between weak extinction (WE) and strong extinction (SE) animals that did or did not receive DBS were not significant. BLA, basolateral amygdala; CAI, hippocampal subfield; CA3, hippocampal subfield; CgI, cingulate gyrus, area I; Cg2, cingulate gyrus, area 2; Ce, central nucleus of the amygdala; IL, infralimbic cortex; La, lateral nucleus of the amygdala; PL, prelimbic cortex. WE CTL $(n=6)$, WE DBS $(n=6)$, SE CTL $(n=8)$, and SE DBS $(n=8)$. Statistical results may be found in Supplementary Table 2.

$(0.27 \pm 0.05 \mathrm{~Hz}$; median 0.13$)$ had a similar firing rate, values recorded in the former were significantly higher than in DBS-treated WE $(0.2 \pm 0.04 \mathrm{~Hz}$; median 0.07 ; mean rank diff. 27.92) and SE animals $(0.12 \pm 0.03 \mathrm{~Hz}$; median 0.06 ; mean rank diff. 38.87).

\section{DISCUSSION}

The present results suggest that the chronic administration of IL DBS to WE rats improves extinction deficits, anxietylike behavior, and reduces BLA cell firing.

Previous studies found that IL stimulation tightly coupled with the CS (Milad et al, 2004) or given immediately after the retrieval of fear memory (Maroun et al, 2012) may enhance fear extinction. Because a comparable DBS protocol would not be easily achievable in the clinic, in the first study we tested whether prolonged stimulation delivered during extinction (ie, a scenario that would mimic the administration of DBS to patients during exposure therapy sessions) could enhance extinction learning. The negative results of this experiment suggest that the timeframe for DBS administration in relation to CS may be critical, as previously reported (Milad et al, 2004).

In addition to the studies mentioned above, different preclinical reports have shown improvements in fear conditioning, stress, and anxiety following BLA (Langevin et al, 2010), hippocampus (Garcia et al, 2008), and ventral striatum stimulation (Rodriguez-Romaguera et al, 2012). Though these have greatly contributed to our understanding of mechanisms of fear and stress, they are limited from a translational perspective. First, the models appear to be more suitable to study acute responses rather than persistent behavioral deficits induced by a traumatic experience. Second, in contrast to the chronic use of clinical DBS, only short-term stimulation has been tested in these animal studies (Langevin et al, 2010; Milad et al, 2004; SaldivarGonzalez et al, 2003; Vidal-Gonzalez et al, 2006). Finally, most models rely on group-averaged data and have not taken into account individual differences in stress response. This is 
Table I Correlation between zif268 Expression in the Infralimbic Cortex (IL) and Different Prefrontal Cortex, Amygdala, and Hippocampal Regions

\begin{tabular}{|c|c|c|c|c|}
\hline & WE CTL & SE CTL & WE DBS & SE DBS \\
\hline \multicolumn{5}{|c|}{ Prefrontal cortex } \\
\hline $\mathbb{I L} \times \mathrm{PL}$ & $r=0.72 ; p=0.1$ & $r=0.91 ; p=0.02$ & $r=0.87 ; p=0.002$ & $r=0.94 ; p=0.001$ \\
\hline$|\mathrm{L} \times \mathrm{Cg}|$ & $r=0.21 ; p=0.6$ & $r=0.76 ; p=0.03$ & $r=0.96 ; p=0.001$ & $r=0.95 ; p=0.001$ \\
\hline $\mathrm{IL} \times \mathrm{Cg} 2$ & $r=0.68 ; p=0.1$ & $r=0.65 ; p=0.08$ & $r=0.87 ; p=0.003$ & $r=0.97 ; p=0.001$ \\
\hline $\mathrm{IL} \times \mathrm{BLA}$ & $r=-0.32 ; p=0.5$ & $r=0.32 ; p=0.04$ & $r=0.62 ; p=0.2$ & $r=0.47 ; p=0.3$ \\
\hline $\mathrm{IL} \times \mathrm{Ce}$ & $r=0.37 ; p=0.5$ & $r=0.70 ; p=0.05$ & $r=0.86 ; p=0.004$ & $r=0.62 ; p=0.1$ \\
\hline \multicolumn{5}{|c|}{ Hippocampus } \\
\hline
\end{tabular}

BLA, basolateral amygdala; CAI, hippocampal subfield; CA3, hippocampal subfield; CgI, cingulate gyrus, area I; Cg2, cingulate gyrus, area 2; Ce, central nucleus of the amygdala; DBS, deep brain stimulation; DG, dentate gyrus; La, lateral nucleus of the amygdala; PL, prelimbic cortex; SE, strong extinction; WE, weak extinction.

a

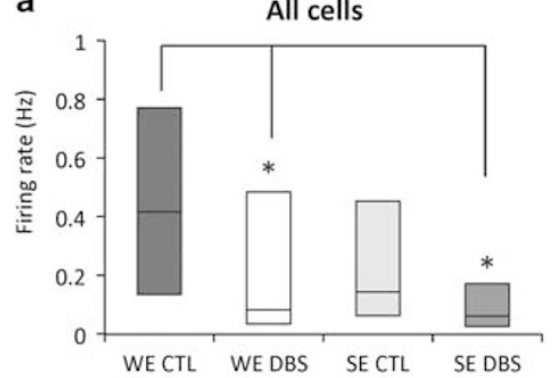

C

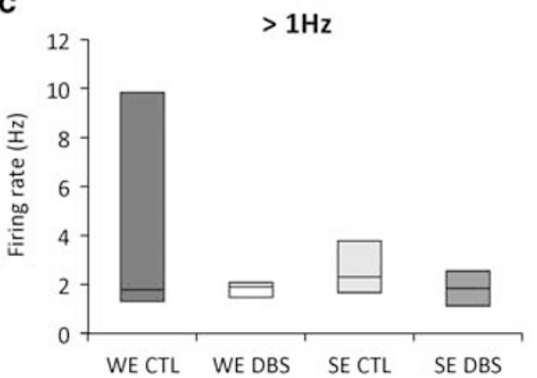

b

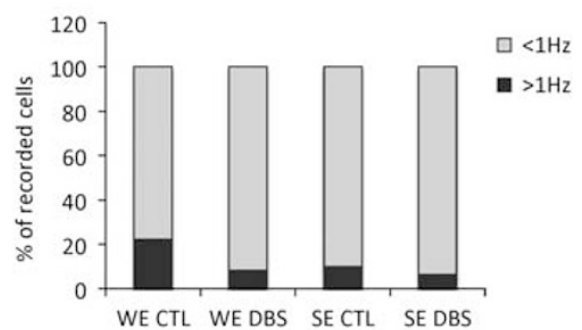

d

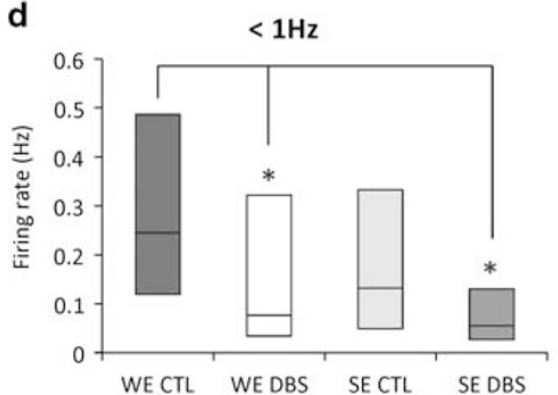

Figure 4 Chronic deep brain stimulation (DBS) reduces basolateral amygdala (BLA) cell firing in weak extinction rats (WE). (a) In vivo electrophysiological recordings of BLA cells in anesthetized rats showed that when all cells were compared, BLA neurons in WE CTL fired at higher rates than in WE DBS, SE DBS, and, to a lesser extent, SE CTL groups. (b) In WE CTL rats, 22\% of BLA cells fired > I Hz. This proportion was 2-3 times higher than in SE CTL, WE DBS, and SE DBS groups $(p=0.29)$. (c) In WE CTL, the firing of $>$ I Hz cells was twice as high as in SE CTLs, WE DBS, and SE DBS groups, though values did not reach statistical significance $(p=0.97)$. (d) The analysis of BLA cell firing $<I \mathrm{~Hz} B L A$ (ie, putative principal cells) yielded significant results across groups ( $p<0.0 \mathrm{I}$ ). Although WE CTL and SE CTL had a similar firing rate, values recorded in the former group were significantly higher than in DBS-treated WE and SE animals. Graphs represent median and quartiles. * $P \leqslant 0.05$ compared with WE CTL.

fundamentally different from the human scenario where exposure to a traumatic event generates an acute stress response in most individuals, of whom only a percentage will develop PTSD. Considering these limitations, we investigated the effects of chronic IL stimulation in WE rats. Overall, we found that DBS not only improved extinction deficits but also anxiety-like behavior in this group of animals.
DBS has been suggested to elicit its therapeutic effects by inhibiting local neuronal populations (Florence et al, 2015; Hamani and Temel, 2012). This is unlikely to be the mechanism of action in this animal model, as lesions and pharmacological inactivation of the IL impair rather than improve extinction (Jinks and McGregor, 1997; Laurent and Westbrook, 2009; Morawska and Fendt, 2012; Quirk et al, 
2000). An alternative mechanism involves the modulation of activity in regions at a distance from the target through the activation of axonal projections near the electrodes (Anderson et al, 2003; Florence et al, 2015; Hamani and Temel, 2012; Hashimoto et al, 2003). To begin addressing this possibility, we examined IEG expression in structures implicated in mechanisms of stress, anxiety, and PTSD, namely the PFC, amygdala, and hippocampus. Except for a general DBS effect on CA1, no major differences in zif268 expression were found when groups of SE and WE animals that did or did not receive DBS were compared. This is in contrast to our previous studies in which acute PFC DBS (delivered for a few hours) was shown to increase IEG expression in different cortical and subcortical structures (Hamani et al, 2014). Such discrepancies may be explained by the chronic nature of DBS delivery in the present work that may have favored the development of compensatory mechanisms to equalize zif268 mRNA levels across groups.

In addition to studying zif268 expression in individual brain regions, we have also examined correlations between IEG expression in the IL and PFC, amygdala, and hippocampus. Our results suggest that IEG correlations between the target zone and the BLA in particular were disrupted in WE animals and reestablished after chronic DBS treatment.

To better appraise the consequences of delivering chronic IL stimulation to the BLA, we have conducted in vivo electrophysiology experiments. Previous work has shown that under basal conditions, BLA glutamatergic cells are tonically inhibited by local GABAergic interneurons (Rainnie et al, 1991). Enhancing activity of the former or decreasing GABAergic tone in BLA facilitates fear conditioning, impairs extinction, and increases anxiety (Akirav et al, 2006; FelixOrtiz et al, 2016; Ganon-Elazar and Akirav, 2009; Kuhnert et al, 2013; Laurent et al, 2008a; Laurent and Westbrook, $2008 \mathrm{~b})$. Studies in anesthetized rodents have shown that BLA principal cells normally depolarize when PFC neurons fire spontaneous action potentials (Likhtik et al, 2005). This is to be expected as PFC-BLA projections are largely glutamatergic. In contrast, pulses or short trains of vmPFC stimulation, particularly at high frequencies, decrease the firing rate of BLA principal cells (Likhtik et al, 2005; Rosenkranz and Grace, 2001; Rosenkranz et al, 2003). The most commonly accepted mechanism to explain these observations is that trains of high-frequency stimulation recruit BLA interneurons that inhibit principal cells by releasing GABA (Likhtik et al, 2005; Rosenkranz and Grace, 2001; Rosenkranz et al, 2003). Based on these assumptions, our initial hypothesis was that chronic DBS would decrease firing of putative BLA principal cells (ie, firing rate $<1 \mathrm{~Hz}$ ) and increase activity of cells firing at higher rates in WE rats. Although stimulation indeed reduced firing of $<1 \mathrm{~Hz}$ cells in WE animals, it also decreased activity of $>1 \mathrm{~Hz}$ active units. This suggests that DBS induced an overall reduction in BLA cell activity with both putative principal cells and interneurons showing lower firing rates (ie, a pattern that closely resembled the one recorded in SE animals). Whether this is in fact one of the mechanisms responsible for the reduced fear/anxiety-like behavior observed in WE animals receiving DBS remains to be demonstrated. It should also be noted that DBS was not active during the recordings. This suggests that some form of plasticity may have occurred so that BLA cell firing in WE
DBS rats more closely resembled the one observed in SE rather than WE animals.

In summary, our results suggest that DBS applied chronically to the IL of WE rats improves extinction deficits, anxiety-like behavior, reverses abnormal neurocircuitry traits, and reduces BLA cell firing. Altogether, these findings indicate that DBS may be capable of reversing structural deficits in PTSD-like animals, shedding light on the therapeutic mechanism of this treatment.

\section{FUNDING AND DISCLOSURE}

This work was supported in part with funds from the National Institutes of Health (1R21MH110846-01). CH received honoraria from St Jude Medical and Medtronic. The other authors declare no conflict of interest.

\section{REFERENCES}

Akirav I, Raizel H, Maroun M (2006). Enhancement of conditioned fear extinction by infusion of the GABA(A) agonist muscimol into the rat prefrontal cortex and amygdala. Eur J Neurosci 23: 758-764.

Anderson ME, Postupna N, Ruffo M (2003). Effects of highfrequency stimulation in the internal globus pallidus on the activity of thalamic neurons in the awake monkey. J Neurophysiol 89: $1150-1160$.

Breslau N, Davis GC, Andreski P, Peterson E (1991). Traumatic events and posttraumatic stress disorder in an urban population of young adults. Arch Gen Psychiatry 48: 216-222.

Breslau N, Kessler RC, Chilcoat HD, Schultz LR, Davis GC, Andreski P (1998). Trauma and posttraumatic stress disorder in the community: the 1996 Detroit Area Survey of Trauma. Arch Gen Psychiatry 55: 626-632.

Bush DE, Sotres-Bayon F, LeDoux JE (2007). Individual differences in fear: isolating fear reactivity and fear recovery phenotypes. J Trauma Stress 20: 413-422.

Cohen H, Zohar J, Matar MA, Zeev K, Loewenthal U, Richter-Levin G (2004). Setting apart the affected: the use of behavioral criteria in animal models of post traumatic stress disorder. Neuropsychopharmacology 29: 1962-1970.

Creed MC, Hamani C, Nobrega JN (2012). Early gene mapping after deep brain stimulation in a rat model of tardive dyskinesia: comparison with transient local inactivation. Eur Neuropsychopharmacol 22: 506-517.

Fairbank JA, Ebert L, Costello EJ (2000). Epidemiology of traumatic events and post-traumatic stress disorder. In:Nutt D, Davidson JRT, Zohar J (eds) Post Traumatic Stress Disorder: Diagnosis, Management and Treatment. Martin Dunitz: London. pp 17-27.

Felix-Ortiz AC, Burgos-Robles A, Bhagat ND, Leppla CA, Tye KM (2016). Bidirectional modulation of anxiety-related and social behaviors by amygdala projections to the medial prefrontal cortex. Neuroscience 321: 197-209.

Florence G, Sameshima K, Fonoff ET, Hamani C (2015). Deep brain stimulation: more complex than the inhibition of cells and excitation of fibers. Neuroscientist 22: 332-345.

Ganon-Elazar E, Akirav I (2009). Cannabinoid receptor activation in the basolateral amygdala blocks the effects of stress on the conditioning and extinction of inhibitory avoidance. J Neurosci 29: $11078-11088$.

Garcia R, Spennato G, Nilsson-Todd L, Moreau JL, Deschaux O (2008). Hippocampal low-frequency stimulation and chronic mild stress similarly disrupt fear extinction memory in rats. Neurobiol Learn Mem 89: 560-566. 
Hamani C, Amorim BO, Wheeler AL, Diwan M, Driesslein K, Covolan L et al (2014). Deep brain stimulation in rats: different targets induce similar antidepressant-like effects but influence different circuits. Neurobiol Dis 71: 205-214.

Hamani C, Diwan M, Isabella S, Lozano AM, Nobrega JN (2010a). Effects of different stimulation parameters on the antidepressantlike response of medial prefrontal cortex deep brain stimulation in rats. J Psychiatr Res 44: 683-687.

Hamani C, Diwan M, Macedo CE, Brandao ML, Shumake J, Gonzalez-Lima F et al (2010b). Antidepressant-like effects of medial prefrontal cortex deep brain stimulation in rats. Biol Psychiatry 67: 117-124.

Hamani C, Dubiela FP, Soares JC, Shin D, Bittencourt S, Covolan L et al (2010c). Anterior thalamus deep brain stimulation at high current impairs memory in rats. Exp Neurol 225: 154-162.

Hamani C, Machado DC, Hipolide DC, Dubiela FP, Suchecki D, Macedo CE et al (2012a). Deep brain stimulation reverses anhedonic-like behavior in a chronic model of depression: role of serotonin and brain derived neurotrophic factor. Biol Psychiatry 71: 30-35.

Hamani C, Temel Y (2012). Deep brain stimulation for psychiatric disease: contributions and validity of animal models. Sci Transl Med 4: 142rv148.

Hashimoto T, Elder CM, Okun MS, Patrick SK, Vitek JL (2003). Stimulation of the subthalamic nucleus changes the firing pattern of pallidal neurons. J Neurosci 23: 1916-1923.

Jinks AL, McGregor IS (1997). Modulation of anxiety-related behaviours following lesions of the prelimbic or infralimbic cortex in the rat. Brain Res 772: 181-190.

Kessler RC, Sonnega A, Bromet E, Hughes M, Nelson CB (1995). Posttraumatic stress disorder in the National Comorbidity Survey. Arch Gen Psychiatry 52: 1048-1060.

Kuhnert S, Meyer C, Koch M (2013). Involvement of cannabinoid receptors in the amygdala and prefrontal cortex of rats in fear learning, consolidation, retrieval and extinction. Behav Brain Res 250: 274-284.

Langevin JP, De Salles AA, Kosoyan HP, Krahl SE (2010). Deep brain stimulation of the amygdala alleviates post-traumatic stress disorder symptoms in a rat model. J Psychiatr Res 44: 1241-1245.

Langevin JP, Koek RJ, Schwartz HN, Chen JW, Sultzer DL, Mandelkern MA et al (2016). Deep brain stimulation of the basolateral amygdala for treatment-refractory posttraumatic stress disorder. Biol Psychiatry 79: e82-e84.

Laurent V, Marchand AR, Westbrook RF (2008a). The basolateral amygdala is necessary for learning but not relearning extinction of context conditioned fear. Learn Mem 15: 304-314.

Laurent V, Westbrook RF (2008b). Distinct contributions of the basolateral amygdala and the medial prefrontal cortex to learning and relearning extinction of context conditioned fear. Learn Mem 15: 657-666.

Laurent V, Westbrook RF (2009). Inactivation of the infralimbic but not the prelimbic cortex impairs consolidation and retrieval of fear extinction. Learn Mem 16: 520-529.

Likhtik E, Pelletier JG, Paz R, Pare D (2005). Prefrontal control of the amygdala. J Neurosci 25: 7429-7437.

Likhtik E, Pelletier JG, Popescu AT, Pare D (2006). Identification of basolateral amygdala projection cells and interneurons using extracellular recordings. J Neurophysiol 96: 3257-3265.

Maren S (2003). The amygdala, synaptic plasticity, and fear memory. Ann NY Acad Sci 985: 106-113.
Maren S, Quirk GJ (2004). Neuronal signalling of fear memory. Nat Rev Neurosci 5: 844-852.

Maroun M, Kavushansky A, Holmes A, Wellman C, Motanis H (2012). Enhanced extinction of aversive memories by highfrequency stimulation of the rat infralimbic cortex. PLOS ONE 7: e35853.

Milad MR, Quirk GJ (2002). Neurons in medial prefrontal cortex signal memory for fear extinction. Nature 420: 70-74.

Milad MR, Vidal-Gonzalez I, Quirk GJ (2004). Electrical stimulation of medial prefrontal cortex reduces conditioned fear in a temporally specific manner. Behav Neurosci 118: 389-394.

Morawska MM, Fendt M (2012). The effects of muscimol and AMN082 injections into the medial prefrontal cortex on the expression and extinction of conditioned fear in mice. J Exp Biol 215(Pt 8): 1394-1398.

Paxinos G, Watson C (1988). The Rat Brain in Stereotaxic Coordinates. Academic Press: San Diego.

Quirk GJ, Russo GK, Barron JL, Lebron K (2000). The role of ventromedial prefrontal cortex in the recovery of extinguished fear. J Neurosci 20: 6225-6231.

Rainnie DG, Asprodini EK, Shinnick-Gallagher P (1991). Inhibitory transmission in the basolateral amygdala. J Neurophysiol 66: 999-1009.

Reznikov R, Binko M, Nobrega JN, Hamani C (2016). Deep brain stimulation in animal models of fear, anxiety, and posttraumatic stress disorder. Neuropsychopharmacology 41: 2810-2817.

Reznikov R, Diwan M, Nobrega JN, Hamani C (2015). Towards a better preclinical model of PTSD: characterizing animals with weak extinction, maladaptive stress responses and low plasma corticosterone. J Psychiatr Res 61: 158-165.

Rodriguez-Romaguera J, Do Monte FH, Quirk GJ (2012). Deep brain stimulation of the ventral striatum enhances extinction of conditioned fear. Proc Natl Acad Sci USA 109: 8764-8769.

Rosenkranz JA, Grace AA (1999). Modulation of basolateral amygdala neuronal firing and afferent drive by dopamine receptor activation in vivo. J Neurosci 19: 11027-11039.

Rosenkranz JA, Grace AA (2001). Dopamine attenuates prefrontal cortical suppression of sensory inputs to the basolateral amygdala of rats. J Neurosci 21: 4090-4103.

Rosenkranz JA, Moore H, Grace AA (2003). The prefrontal cortex regulates lateral amygdala neuronal plasticity and responses to previously conditioned stimuli. J Neurosci 23: 11054-11064.

Saldivar-Gonzalez JA, Posadas-Andrews A, Rodriguez R, Gomez C, Hernandez-Manjarrez ME, Ortiz-Leon S et al (2003). Effect of electrical stimulation of the baso-lateral amygdala nucleus on defensive burying shock probe test and elevated plus maze in rats. Life Sci 72: 819-829.

Sierra-Mercado D, Padilla-Coreano N, Quirk GJ (2011). Dissociable roles of prelimbic and infralimbic cortices, ventral hippocampus, and basolateral amygdala in the expression and extinction of conditioned fear. Neuropsychopharmacology 36: $529-538$.

Vidal-Gonzalez I, Vidal-Gonzalez B, Rauch SL, Quirk GJ (2006). Microstimulation reveals opposing influences of prelimbic and infralimbic cortex on the expression of conditioned fear. Learn Mem 13: 728-733.

Yehuda R, Southwick SM, Nussbaum G, Wahby V, Giller EL Jr, Mason JW (1990). Low urinary cortisol excretion in patients with posttraumatic stress disorder. J Nerv Ment Dis 178: $366-369$.

Supplementary Information accompanies the paper on the Neuropsychopharmacology website (http://www.nature.com/npp) 